Acta Crystallographica Section D

Biological

Crystallography

ISSN 0907-4449

\section{MAD data collection - current trends}

\section{Martin A. Walsh, ${ }^{\mathrm{a} *}+$ Gwyndaf Evans, ${ }^{\mathrm{a}} \mp$ Ruslan Sanishvili, ${ }^{\mathrm{a}}$ Irene Dementieva $^{a}$ and Andrzej Joachimiak $^{a, b}$}

\begin{abstract}
a Bioscience Division/Structural Biology Center, Argonne National Laboratory, 9700 S. Cass Ave, Argonne IL 60439, USA, and ${ }^{\mathbf{b}}$ Northwestern University, Department of Biochemistry, Molecular Biology and Cell Biology, Evanston IL 60208, USA
\end{abstract}

+ Present address: IRBM P. Angeletti, Via Pontina km 30.600, 00040 Pomezia (Rome), Italy.

₹ Present address: MRC Laboratory of Molecular Biology, Hills Road, Cambridge CB2 2QH, England.

Correspondence e-mail: walsh@irbm.it
The multiwavelength anomalous dispersion (MAD) method of protein structure determination is becoming a routine technique in protein crystallography. The increased number of wavelength-tuneable synchrotron beamlines capable of performing challenging MAD experiments, coupled with the widespread availability of charge-coupled device (CCD) based X-ray detectors with fast read-out times have brought MAD structure determination to a new exciting level. Ultrafast MAD data collection is now possible and, with the widespread use of selenium in the form of selenomethionine for phase determination, the method is growing in popularity. Recent developments in crystallographic software are complementing the above advances, paving the way for rapid protein structure determination. An overview of a typical MAD experiment is described, with emphasis on the rates and quality of data acquisition now achievable at third-generation synchrotron sources.

\section{Conventions and abbreviations}

Typically, X-rays are measured in wavelengths in diffraction experiments; here, X-rays described in wavelength units and energy in electron volts are interchanged, as it is more logical to describe X-rays in units of energy with respect to spectroscopy measurements. The conversion factor between electron volts and angstroms is

$$
E(\mathrm{keV})=12.3985 / \lambda(\AA) .
$$

APS, Advanced Photon Source, USA; CHESS, Cornell High Energy Synchrotron Source, USA; ESRF, European Synchrotron Radiation Facility, France; NSLS, National Synchrotron Light Source, USA; SSRL, Standford Synchrotron Radiation Laboratory, USA; SRS, Synchrotron Radiation Source, UK; Se-Met, selenomethionine.

\section{Introduction}

In the past two decades, MAD has grown from slow beginnings to a stage where it rivals multiple isomorphous replacement (MIR) as the method of choice for solving protein crystal structures (Hendrickson \& Ogata, 1997). Although the method relies on a small anomalous signal, it has a number of advantages over the MIR method, in that all data can be measured from a single crystal and accurate phase information is obtainable. A number of excellent reviews on the MAD technique, which cover all aspects of the method in greater detail are available (Hendrickson, 1991; Hendrickson \& Ogata, 1997; Smith, 1991).
Received 26 March 1999 Accepted 21 June 1999
(C) 1999 International Union of Crystallography Printed in Denmark - all rights reserved 
The majority of the problems which have restricted the growth of MAD in the past have now been solved. These have included formulation of the underlying theory, construction of the necessary instrumentation to perform an experiment and the development of user-friendly software which uses the anomalous diffraction data to abstract phases (Ogata, 1998). Two of the most important advances for the success of the method have been the now-routine method of crystal freezing at liquid-nitrogen temperatures (Braig et al., 1994; Garman \& Schneider, 1997; Rodgers, 1997) and the slow but steady rise in the availability of synchrotron beamlines dedicated to MAD. These beamlines have been designed to meet the stringent experimental criteria laid down by the method, such as accurate and reproducible wavelength tunability and stability (Evans \& Pettifer, 1996) and accurate measurement of diffraction intensities. Cryofreezing is important, as crystal lifetime is significantly increased. This creates the possibility of collecting the several high-quality data sets from a single crystal which are typically required for a MAD experiment (although cryofreezing of protein crystals does not completely eliminate radiation decay). The resulting isomorphic nature of the data is an important and critical factor when utilizing weak anomalous signals. Furthermore, as the anomalous scattering signal is usually very small, use of only one or two crystals to complete a MAD experiment minimize other sources of systematic errors which remain after scaling and can be detrimental to the success of the experiment. The appearance of dedicated beamlines, for example, X4A and X12C at the NSLS, CHESS F2, SSRL 1-5, SRS 9.5, BM14 ESRF, and in particular the use of thirdgeneration undulator sources at the APS and in the near future at SPring-8 (Japan) and ESRF has resulted in a large increase in the number of MAD experiments being successfully performed (Hendrickson \& Ogata, 1997; Ogata, 1998). Moreover, undulator beamlines provide X-rays of high brilliance, of stable energy and with high-energy resolution with a wide X-ray energy range; these are pre-requisites for a MAD experiment.

These facts, combined with fast read-out detectors, have reduced the time required to complete a MAD experiment from a couple of days to a couple of hours. In this paper, we outline the steps required to collect MAD data and highlight some of the recent results obtained with the current technology.

\section{Background}

\subsection{Overview}

Generally, in protein crystallographic X-ray diffraction experiments it is assumed that the interaction of X-rays with electrons gives rise to elastic scattering of the incident radiation. However, when the energy of the incident X-rays is close to that of an electronic transition in a bound atomic orbital, resonance occurs which results in what is referred to as anomalous scattering. Therefore, the total scattering factor is usually written

$$
f=f_{o}+f^{\prime}+\iota f^{\prime \prime}
$$

where $f$ is the total scattering, $f_{o}$ is the normal or Thomson scattering and $f^{\prime}$ and $\iota f^{\prime \prime}$ are the real and imaginary components of the anomalous scattering. The magnitude of the anomalous scattering is essentially independent of the scattering angle, but does depend strikingly on the energy of the incident radiation. The intensity of the normal scattering, on the other hand, falls off with increasing scattering angle.

Absorption of an X-ray photon provides the energy for an electronic transition, which perturbs the amplitude and phase of the diffracted X-rays and gives rise to anomalous scattering. The characteristic energy for a specific transition is referred to as an absorption edge and can be easily characterized by X-ray absorption spectroscopy. The absorption edges are named with respect to the atomic orbital from which they originate. Thus, electronic transitions which originate from $1 s$ orbitals are referred to as $K$ edges, from $2 p$ orbitals, $L$ edges and from $3 d$ orbitals, $M$ edges. For the atoms $\mathrm{C}, \mathrm{N}, \mathrm{O}$ and $\mathrm{S}$, the magnitude of the anomalous signal is negligible and usually ignored. However, anomalous scattering from sulfur has been successfully used to obtain phase information in a number of cases (Hendrickson \& Teeter, 1981; Dauter et al., 1999). Anomalous scattering effects are more easily detectable for heavier atoms. For non-centrosymmetric structures, as the imaginary component $\left(f^{\prime \prime}\right)$ of the anomalous scattering is $90^{\circ}$ out of phase to that of the normal scattering, Friedel's law, $\left[I_{(h k l)}=\mathrm{I}_{(\overline{h k l})}\right]$ no longer holds. The resultant intensity differences in the Friedel pairs, now referred to as Bijvoet differences, can be used in a somewhat similar way to MIR to obtain phase information.

Protein crystallography beamlines at synchrotron facilities provide a tuneable source of X-rays in the approximate energy range $25-6 \mathrm{keV}(0.5-2.0 \AA)$. This energy range provides us with the opportunity of tuning the incident X-ray energy to a large number of heavy-atom absorption edges which provide a measurable source of anomalous scattering. These elements are often found naturally in proteins (for example, $\mathrm{Ca}, \mathrm{Mn}, \mathrm{Fe}$, $\mathrm{Co}, \mathrm{Cu}, \mathrm{Zn}, \mathrm{Mo}$ ) or can be incorporated into protein structures ( $\mathrm{Se}, \mathrm{Br}, \mathrm{Kr}$, lanthanides, $\mathrm{Hg}$, to name a few, and many other heavy atoms). For a comprehensive list of accessible absorption edges, see Table 1 of the review by Hendrickson \& Ogata (1997). The choice of heavy atom used for a MAD experiment depends on the problem being pursued. For example, in the case of a metalloprotein, no heavy-atom derivative may be necessary (Guss et al., 1988; Hendrickson et al., 1988; Than et al., 1997). It is generally preferable to have a smaller number of anomalous scatterers with a large signal rather than a large number of weak anomalous scatterers. Therefore, the use of heavier atoms, such as one of the lanthanides, is advantageous (Helliwell, 1992). However, the success of the selenomethione MAD approach (Doublié, 1997; Hendrickson et al., 1990), where methionine is substituted for selenomethionine and the selenium used as a source of anomalous scattering, has made this element by far the most popular for use in the MAD technique (Ogata, 1998). The method is widely applicable, as methionine naturally occurs in proteins at about a level of $2 \%$. 
Most Se-Met protein substituted proteins are structurally isomorphous to the native protein. Furthermore, both bacteria and eukaryotic cells can grow on media containing Se-Met, which makes a large number of proteins routinely accessible to the method.

\section{Experimental procedures}

The strategy and rational design of a MAD experiment depends on a number of factors, ranging from the type of anomalous scatter used to the properties of the crystal and the experimental setup. A general description of how to perform a MAD experiment and some of the pitfalls encountered follows.

\subsection{Determination of wavelengths to use}

It is essential in a MAD experiment to maximize the anomalous scattering signal from an incorporated or existing heavy element. Thus, wavelengths must be carefully selected to maximize the $f^{\prime \prime}$ component of the anomalous scattering and as much as possible produce large differences in the $f^{\prime}$ component. In other words, maximize $\Delta f^{\prime}$, which is the source of isomorphous differences between the data collected at different X-ray wavelengths. In order to do this, data typically need to be collected at three different X-ray wavelengths or energies, but only two wavelengths are required to unambiguously assign the phase. Assessments of the quality of the final electron-density maps phased on data ranging from two to five wavelength combinations and data-collection strategies have been dealt with in more detail elsewhere (Evans \& Wilson, 1999; Peterson et al., 1996).

Although both $f^{\prime}$ and $f^{\prime \prime}$ values can be calculated theoretically for isolated atoms in vacuum, these magnitudes do not necessarily represent their true values at the absorption edge of the heavy atom in question when bound to other atomic species. Indeed, the prominent so called 'white-line' feature observed at the absorption-edge threshold of heavy metals (especially for $L_{\mathrm{II}}$ and $L_{\mathrm{III}}$ edges) is not accounted for by theoretical calculations (Cromer \& Libermann, 1970). Both the form and the position of the absorption edge is influenced by the chemical environment of the heavy atom in the protein. Thus, it is imperative to determine the values of the scattering factors experimentally. This can be achieved by measuring an X-ray absorption spectrum from the protein crystal containing the anomalous scattering element. From these data, the precise wavelength/energy of the absorption edge is determined. A typical absorption spectrum measured from an Se-Met-substituted protein crystal is shown in Fig. 1(a).

The $f^{\prime \prime}$ component is directly proportional to the X-ray absorption spectrum, and the $f^{\prime}$ component can be calculated from the $f^{\prime \prime}$ values using the Kramers-Kronig transformation (Hoyt et al., 1984). The program CHOOCH (Evans, 1998) provides a fast and convenient way to extract all the essential information from the X-ray absorption spectrum, i.e. $f^{\prime}$ and $f^{\prime \prime}$ values, the wavelength of the absorption-edge peak and the inflection point (Fig. 1b).
Collecting diffraction data at the absorption-edge peak or 'white line' maximizes the $f^{\prime \prime}$ component of the anomalous scattering (Fig. 1) and produces the largest differences in intensities between Bivjoet mates. A large contrast in $f^{\prime}$ values can be achieved intuitively by measuring data at the inflection point of the absorption edge, minimizing the $f^{\prime}$ component, and at a wavelength remote from the absorption edge, therefore maximizing $\Delta f^{\prime}$. Usually, this so-called 'remote' wavelength data set is collected above the absorption edge (i.e. at lower wavelength/higher energy), where use can still be made of the albeit smaller $f^{\prime \prime}$ value when compared with the absorption edge. In the past, time permitting, it was normal to collect additional data [for example, a remote data set below the absorption edge (higher wavelength/lower energy)] for use in overdetermination of the phasing parameters. This latter

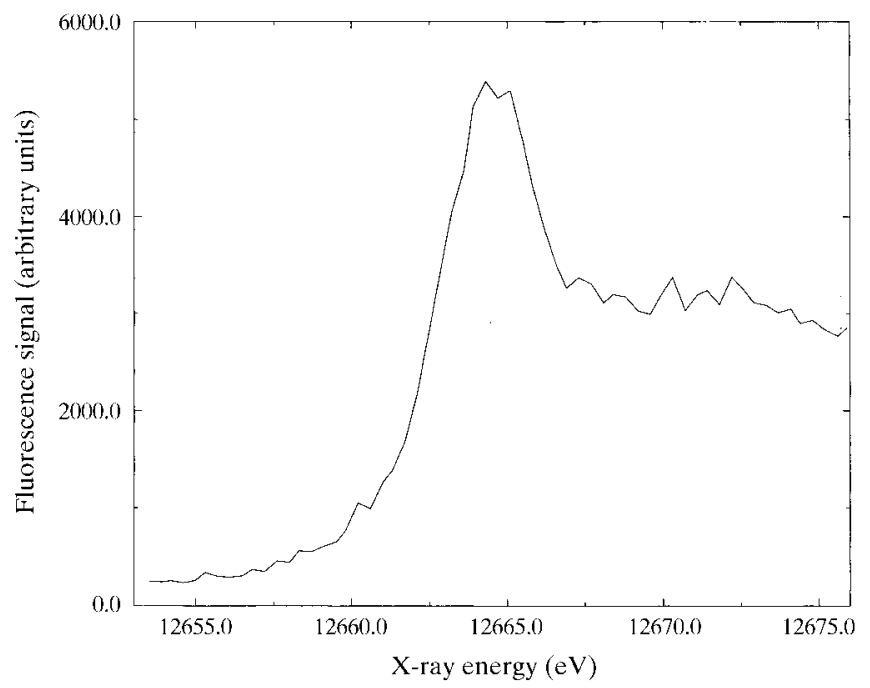

(a)

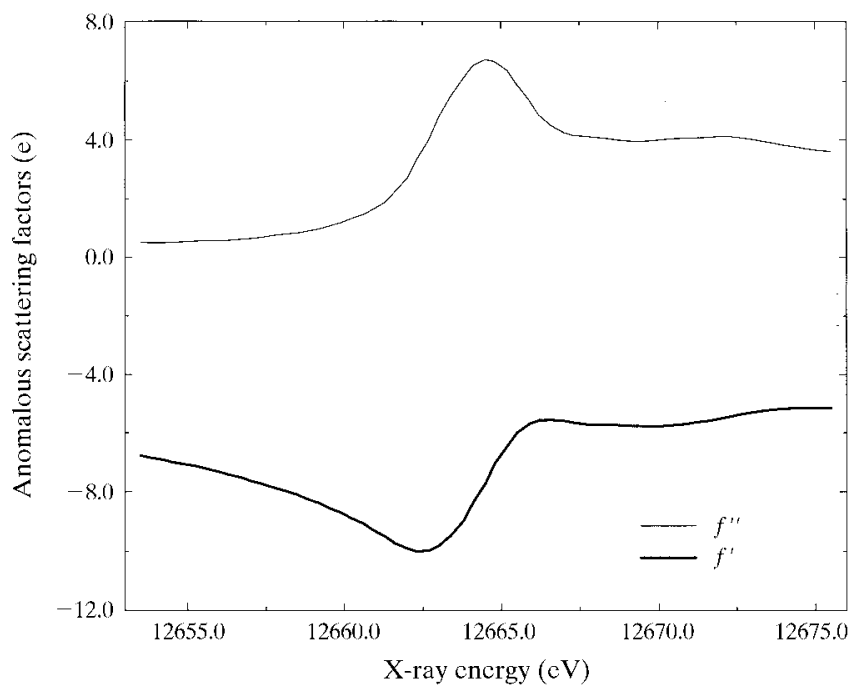

(b)

Figure 1

(a) An X-ray absorption spectrum obtained from a Se-Met substituted protein. The spectrum was measured at the Structural Biology Center undulator beamline 19ID at the APS. (b) Anomalous scattering curves derived using the program $\mathrm{CHOOCH}$ from the data obtained in $(a)$. 
philosophy can be extended to include additional data sets at other wavelengths, although more recent experiments suggest that it is far more useful to collect more redundant data at the minimum two or three wavelengths required for a MAD experiment (Evans \& Wilson, 1999).

The measurement of an X-ray fluorescence spectrum which is representative of all the Se atoms in the protein crystal can be influenced by two effects. The first is oxidation of Se-Met in the protein crystal and the second is anisotropy of Se absorption in Se-Met around its $K$ absorption edge (Hendrickson et al., 1989). The latter is more likely when there is only a small number of Se-Met residues, as it is a result of the $\mathrm{C}-\mathrm{Se}-\mathrm{C}$ planes of the Se-Met residues being aligned approximately parallel to each other. Anisotropy in the Se $K$ edge can be quite striking, resulting in the loss or weakening of the typically well defined absorption peak of the Se $K$ edge, in addition to inducing a shift in energy of the absorption edge which is dependent on the crystal's orientation. These effects can be additionally compounded by the absorption effects in the crystal, as selenium concentrations in protein crystals can exceed $100 \mathrm{mM}$. Oxidation of selenium can also produce poor spectra owing to mixtures of selenium oxidation states. This can usually be avoided by handling Se-Met proteins in a reducing environment. Generally, this entails use of deaerated buffers containing dithiothreitol (DTT) at a concentration of 5-20 $\mathrm{m} M$ during purification and crystallization (Doublié, 1997). The oxidation of Se-Met proteins has been studied in more detail by Smith \& Thompson (1998). Finally, the exact position of the absorption peak may differ slightly because of errors in energy calibration. Therefore, it is always crucial to record the absorption spectrum prior to commencing a MAD experiment. Furthermore, it is possible to employ a device that allows X-ray energy stabilization, even during an X-ray exposure (Evans \& Pettifer, 1996). This is particularly useful where a MAD experiment spans a long period of time, where problems with beam instability and energy drifts are more likely.

\subsection{Data-collection strategies}

In many cases, the anomalous scattering signal is small, usually $2-5 \%$ of the total scattering, and special care must be taken to collect 'the best' MAD data. In order to reduce systematic errors in data collection from absorption, crystal decay and detector non-uniformity of response to X-ray photons, which all may interfere with the precise measurement of the Bijvoet and dispersive differences, two main data-collection protocols have been devised. The first involves aligning the crystal such that Friedel pairs are measured on the same diffraction image. This is aided by use of, in particular, a mini- $\kappa$ goniostat at the Structural Biology Center's (SBC) 19ID undulator beamline at the APS (Rosenbaum \& Westbrook, 1997). The goniostat allows a substantial range of positioning of the mounted crystal, while avoiding loss of data on the detector from the diffracted X-rays being obstructed by the goniostat frame. A disadvantage of aligning a crystal is that data completeness will be somewhat affected by the blind region (Dauter, 1997). If it is not possible to align a crystal along a symmetry axis, the other alternative is to use the inversebeam method. This involves recording Friedel mates on two separate images, usually by collecting a small wedge of data and then rotating the crystal by $180^{\circ}$ around the spindle axis of the goniostat and collecting the same wedge of data. Care is needed to ensure exact centering of the crystal and that the total diffracting volume of the crystal is fully illuminated by the incident X-ray beam. For single anomalous diffraction (SAD) data collected on a home source, the latter technique is indispensable for achieving successful results. The inverse-beam method is mainly intended to measure Bijvoet differences as accurately as possible, but the multiplicity of the data is concurrently doubled. Collecting redundant data is an important aspect of any MAD experiment. Another strategy which can substantially improve the quality of MAD data is reorienting of the crystal to collect data around another crystal axis (Evans, 1993). This again increases the redundancy of the data but, more importantly, it improves the scaling of the data, which is a crucial factor in a MAD experiment. Use of a mini- $\kappa$ goniostat makes the latter strategy possible. In most cases, cryofreezing of protein crystals allows all data to be collected from a single crystal, again reducing systematic errors in data collection. If the crystal is radiation-sensitive, collecting wedges of data $\left(\right.$ e.g. $\left.10^{\circ}\right)$ successively at each wavelength may be necessary for success. In this way, all data required to determine the phase of a reflection are measured over a short period of time and from the same crystal.

Other considerations to take into account include collecting strong data to reduce statistical errors in the data collection without compromising low-resolution data through excess numbers of overloaded reflections. However, it is important to point out that MAD experiments performed with randomly oriented crystals and using a single pass at each wavelength have been successful at the SBC 19ID beamline (Li et al., 1998; Barycki et al., 1999; Zhang et al., 1999) and at other facilities e.g. BM14 at ESRF (van Montfort et al., 1998). In cases where the technique is being pushed to its limit and the anomalous scattering signal is extremely small, the above strategies can be essential to the success of the experiment.

\section{Recent experiences}

Until recently, the most difficult tasks related to performing a MAD experiment were finding a synchrotron beamline which met the demands of the experiment and then obtaining sufficient time to actually complete the data collection. A dramatic reduction in the time required to carry out a MAD experiment practically avoids problems which can be incurred through beam instability and beam dumps. These can give rise to systematic errors in the data which can lead to the failure of a MAD experiment. Also, all data are collected in close proximity in time and under essentially the same conditions.

Competition for beam time is high, therefore time is a critical parameter in any data collection at a synchrotron, 
particularly for MIR and MAD experiments. Owing to the multiple data sets required in the MAD method, a successful experiment has typically been taking $1-2 \mathrm{~d}$ to complete even
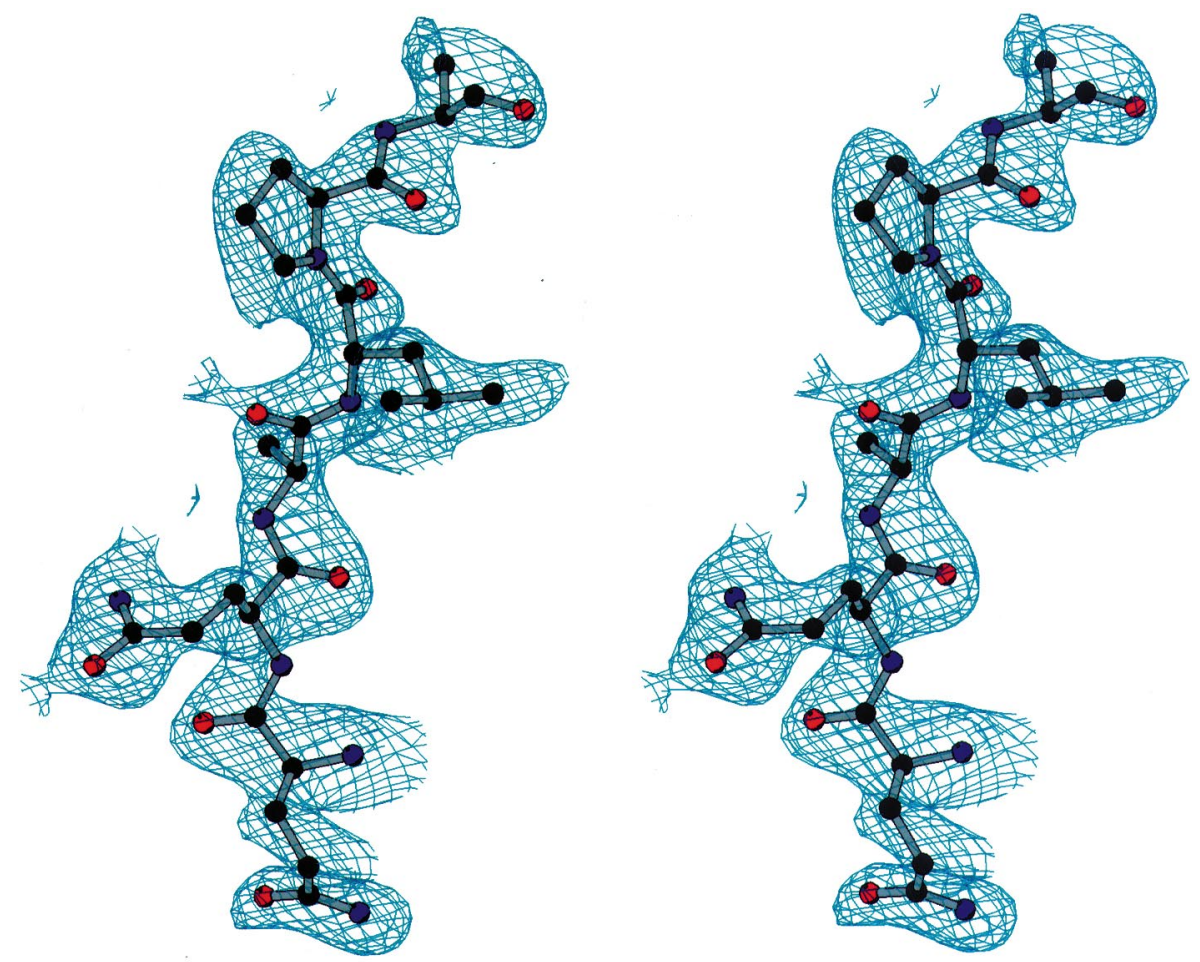

(a)
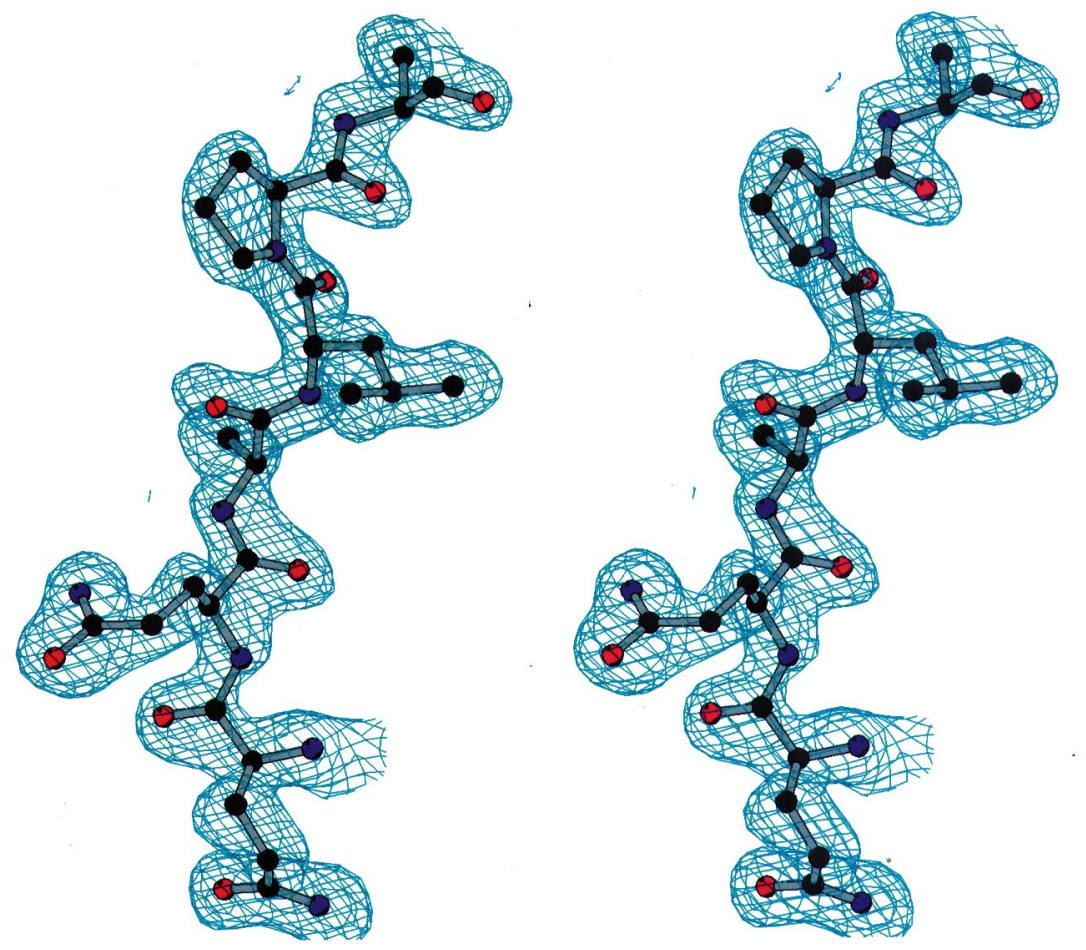

(b)

\section{Figure 2}

A section of the electron-density map of the $170 \mathrm{kDa}$ protein (see text) ( $a$ ) based solely on MAD phasing information at $2.4 \AA$ resolution and $(b)$ after density modification and phase extension to $1.65 \AA$ with the program $D M$ (Collaborative Computational Project, Number 4, 1994). The figure was produced with the program BOBSCRIPT (Esnouf, 1997; Kraulis, 1991) at third-generation synchrotron sources (Smith et al., 1996). Drastic reductions in the time needed to collect complete MAD data have been observed at the SBC 19ID undulator beamline at the APS and at other facilities with similar configurations; for example, BM14 at ESRF, a bendingmagnet beamline dedicated to MAD experiments (ESRF, 1998). These results have gone far beyond the recent expectations in the field. A brief description of two experiments at the SBC undulator beamline 19ID highlight what we can expect to see in the near future at thirdgeneration synchrotron sources (Helliwell, 1998).

\subsection{Two examples of Se-Met MAD data-collection experiments}

The SBC 19ID undulator beamline at the APS situated at Argonne National Laboratory provides a stable source of X-rays of high brilliance with highenergy resolution and rapid reproducible tuning, ideal for performing a MAD experiment. The beamline is equipped with a large area mosaic $3 \times 3$ CCD detector with a fast read out (Westbrook \& Naday, 1997). Using this type of configuration, complete MAD data sets are being collected routinely in less than $1 \mathrm{~h}$ (Walsh, Dementieva et al., 1999; Li et al., 1998; Barycki et al., 1999; Zhang et al., 1999).

The first MAD experiment performed at this beamline with the above configuration was carried out on an approximately $16 \mathrm{kDa}$ molecular-weight protein (peptide-binding domain of a thermophilic chaperonin), which crystallized in the orthorhombic space group $C 222_{1}$ with unit-cell dimensions $a=62.7$, $b=64.7, c=74.2 \AA$. The protein has three methionines in its amino-acid sequence. Therefore, substitution of these methionines with selenomethionine provided a good measurable source of anomalous scattering. When anomalous scattering factors are known, an estimate of the maximum anomalous $\left(2 f_{\max }^{\prime \prime}\right)$ and dispersive $\left(\left|f_{\lambda i}^{\prime}-f_{\lambda j}^{\prime}\right|_{\text {max }}\right)$ signals as a fraction of the total scattering of the protein $\left(\left\langle\left|F_{p}\right|\right\rangle\right)$ can be obtained (Smith, 1991). For proteins, a rough estimate of $\left\langle\left|F_{p}\right|\right\rangle$ is $(346 \times$ the number of amino acids $)^{1 / 2}$. The estimates are then made by 
maximum anomalous signal $=\left(N_{\mathrm{ano}} / 2\right)^{1 / 2} \times 2 f_{\max }^{\prime \prime} /\left\langle\left|F_{p}\right|\right\rangle$ maximum dispersive signal $=\left(N_{\text {ano }} / 2\right)^{1 / 2}\left|f_{\lambda i}^{\prime}-f_{\lambda j}^{\prime}\right|_{\max } /\left\langle\left|F_{p}\right|\right\rangle$,

where $N_{\text {ano }}$ is the number of anomalous scatterers and $\lambda_{i}$ is the inflection point data $\left(f_{\min }^{\prime}\right)$ and $\lambda_{j}$ a remote wavelength data set.

The expected anomalous signal in this case, was approximately $6 \%$ of the total scattering of the crystal. The crystals diffract strongly to $2.0 \AA$ and are relatively stable to $\mathrm{X}$-radiation. Therefore, this was an ideal test experiment for assessing the capability of the beamline for collecting MAD data. A conventional four-wavelength MAD experiment was performed, with data being collected to $2.25 \AA$ resolution at the absorption-edge peak, the absorption-edge inflection point and two remote energies, one on each side of the absorption edge. The crystal was approximately aligned so as to record Bijvoet pairs simultaneously on the same image. Data sets were collected in a single pass with a $2^{\circ}$ oscillation and $3 \mathrm{~s}$ exposure per frame. A total of $120^{\circ}$ of data were collected for each independent wavelength. The total datacollection time for the experiment was $23 \mathrm{~min}$. Taking into account the time required to mount and orient the crystal and obtain an X-ray fluorescence spectrum, the complete experiment took 45 min to perform (Walsh, Dementieva et al., 1999). Independent analyses of these data with the program suites CCP4 (Collaborative Computational Project, Number 4, 1994) and CNS (Brunger et al., 1998) proceeded smoothly and produced electron-density maps which were fully interpretable and of very high quality.

A second far more challenging experiment was carried out later at the beamline, using the same general philosophy as the first successful MAD experiment summarized above. In this case the protein under study crystallizes as a homodecamer in the triclinic space group $P 1$ (Walsh, Otwinowski et al., 1999). The molecular weight of the monomer subunit is $17 \mathrm{kDa}$, giving a total of $170 \mathrm{kDa}$ in the asymmetric unit, with a total of 40 selenomethionine residues for use in phasing. Se-Met incorporation was achieved by inhibition of methionine biosynthesis (Van Duyne et al., 1993), which resulted in only $83 \%$ incorporation as measured by mass spectrometry (WM Keck Foundation, Biotechnology Resource Laboratory, Yale University). Again, a four-wavelength MAD experiment was performed. A total of $360^{\circ}$ of data were collected in a single pass at each wavelength, using a $1^{\circ}$ oscillation and $3 \mathrm{~s}$ exposure per frame. The resolution of the data collected was $2.4 \AA$. In this case, the total time required to collect the X-ray data was $127 \mathrm{~min}$. The total time required to carry out the experiment was just $2.5 \mathrm{~h}$. The data were again of high quality, and determination of the sub-structure of the anomalous scattering centers was facilitated with new software recently available for locating high numbers of heavy atoms both in the $C C P 4$ suite (program RANTAN) and a new automatic heavyatom search program in the CNS suite. After the MAD experiment was completed, a high-resolution ( $1.65 \AA$ ) data set was collected after translating a new section of the crystal into the X-ray beam at $12 \mathrm{keV}(1.0332 \AA)$. Solvent flattening and phase extension against these high-resolution data produced experimental maps of excellent quality (Fig. 2). These phases were then input into the program $A R P / w A R P 5.0$, which contains an auto-tracing module (Perrakis et al., 1999). The program successfully traced the polypeptide chains of all ten subunits, in addition to modeling over $70 \%$ of the 1156 aminoacid side chains; these results were achieved without employing any non-crystallographic averaging. Thus, a combination of fast data-acquisition times and analysis, followed by the use of automated model building, significantly reduced the time required to obtain a reliable three-dimensional model of the protein. The ARP/wARP 5.0 program can produce useful results with diffraction data extending to a resolution of $2.3 \AA$ or higher. The program is sure to become an important tool for building initial protein models (Perrakis et al., 1999).

\section{Conclusions}

The MAD method is now benefiting from the improved facilities available at synchrotron sites around the world and a number of other improvements in the field. This has enabled both rapid and accurate measurements of diffraction data. The wider availability of X-ray beamlines providing X-rays of high flux and stable energy with fast reproducible tuning and highenergy resolution, coupled to fast read-out large-area CCD detectors have made these results possible.

The collection of ultrafast high-quality X-ray diffraction data, whether it be for a MAD experiment or a single-wavelength experiment, at the SBC beamline at the APS and other beamlines with similar facilities around the world (Helliwell, 1998; Hendrickson \& Ogata, 1997) is contributing to substantial reductions in the time required to solve a threedimensional structure of a macromolecule.

The collection of high-quality diffraction data with dramatically reduced data-collection times are now complementing the latest developments in crystallographic software suites for analysis of diffraction data and provide a realistic platform for accelerated protein structure determination.

This project was supported by the US Department of Energy, Office of Health and Environmental Research, under contract W-31-109-Eng-38.

\section{References}

Barycki, J. J., O’Brien, L. K., Bratt, J. M., Zhang, R., Sanishvili, R., Strauss, A. W. \& Banaszak, L. J. (1999). Biochemistry, 38, 5786-5798.

Braig, K., Otwinowski, Z., Hedge, R., Boisvet, D., Joachimiak, A., Horwich, A. L. \& Sigler, P. B. (1994). Nature (London), 371, 578-586.

Brunger, A. T., Adams, P. D., Clore, G. M., DeLano, W. L., Gros, P., Grosse-Kunstleve, R. W., Jiang, J. S., Kuszewski, J., Nilges, M., Pannu, N. S., Read, R. J., Rice, L. M., Simonson, T. \& Warren, G. L. (1998). Acta Cryst. D54, 905-921.

Collaborative Computational Project, Number 4 (1994). Acta Cryst. D50, 760-763.

Cromer, D. T. \& Libermann, D. (1970). J. Chem. Phys. 53, 1891-1898. 
Dauter, Z. (1997). Methods Enzymol. 276, 326-344.

Dauter, Z., Dauter, M., de la Fortelle, E., Bricogne, G. \& Sheldrick, G. M. (1999). J. Mol. Biol. 289, 83-92.

Doublié, S. (1997). Methods Enzymol. 276, 523-530.

Esnouf, R. M. (1997). J. Mol. Graph. 15, 132-134.

ESRF (1998). ESRF Highlights 1997/1998. ESRF, Grenoble, France.

Evans, G. (1998). CHOOCH Download Page. http://lagrange.mrclmb.cam.ac.uk/doc/gwyndaf/chooch.html.

Evans, G. \& Pettifer, R. F. (1996). Rev. Sci. Instrum. 67, 3428-3433.

Evans, G. \& Wilson, K. S. (1999). Acta Cryst. D55, 67-76.

Evans, P. R. (1993). Proceedings of the CCP4 Study Weekend: Data Collection and Processing, edited by L. Sawyer, N. Isaacs \& S. Bailey, pp. 114-122. Warrington: Daresbury Laboratory.

Garman, E. F. \& Schneider, T. R. (1997). J. Appl. Cryst. 30, 211-237.

Guss, J. M., Merritt, E. A., Phizackerley, R. P., Hedman, B., Murata, M., Hodgson, K. O. \& Freeman, H. C. (1988). Science, 241, 806-11.

Helliwell, J. R. (1992): Macromolecular Crystallography with Synchrotron Radiation. Cambridge University Press.

Helliwell, J. R. (1998). Nature Struct. Biol. 5, 614-617.

Hendrickson, W. A. (1991). Science, 254, 51-58.

Hendrickson, W. A., Horton, J. R. \& LeMaster, D. M. (1990). EMBO J. 9, 1665-1672.

Hendrickson, W. A. \& Ogata, C. M. (1997). Methods Enzymol. 276, 494-523.

Hendrickson, W. A., Pähler, A., Smith, J. L., Satow, Y., Merritt, E. A. \& Phizackerley, R. P. (1989). Proc. Natl Acad. Sci. USA, 86, 2190-2194.

Hendrickson, W. A., Smith, J. L., Phizackerley, R. P. \& Merritt, E. A. (1988). Proteins, 4, 77-88.

Hendrickson, W. A. \& Teeter, M. M. (1981). Nature (London), 290, 107-113.

Hoyt, J., de Fontaine, D. \& Warburton, W. (1984). J. Appl. Cryst. 17, 344-351.
Kraulis, P. J. (1991). J. Appl. Cryst. 24, 946-950.

Li, Y., Korolev, S. V. \& Waksman, G. (1998). EMBO J. 17, 7514-25.

Montfort, R. L. M. van, Pijning, T., Kalk, K. H., Hangyi, I., Kouwijzer, M. L. C. E., Robillard, T. G. \& Dijkstra, B. W. (1998). Structure, 6, 377-388.

Ogata, C. M. (1998). Nature Struct. Biol. 5, 638-640.

Perrakis, A., Morris, R. \& Lamzin, V. S. (1999). Nature Struct. Biol. 6, 458-463.

Peterson, M. R., Harrop, S. J., McSweeney, S. M., Leonard, G. A., Thompson, A. W., Hunter, W. N. \& Helliwell, J. R. (1996). J. Synchrotron Rad. 3, 24-34.

Rodgers, D. W. (1997). Methods Enzymol. 276, 183-203.

Rosenbaum, G. \& Westbrook, E. M. (1997). Synchrotron Radiation Instrumentation Tenth National Conference, Ithaka, NY, AIP Conference Proceedings 417, edited by E. Fontes, p. 186. Woodbury, NY: American Institute of Physics.

Smith, J. L. (1991). Curr. Opin. Struct. Biol. 1, 1002-1011.

Smith, J. L. \& Thompson, A. (1998). Structure, 6, 815-819.

Smith, J. L., Thompson, A. \& Ogata, C. M. (1996). Synchrotron Radiat. News, 9, 12-14.

Than, M. E., Hof, P., Huber, R., Bourenkov, G. P., Bartunik, H. D., Buse, G. \& Soulimane, T. (1997). J. Mol. Biol. 271, 629-44.

Van Duyne, G. D., Standaert, R. F., Karplus, P. A., Schreiber, S. L. \& Clardy, J. (1993). J. Mol. Biol. 229, 105-124.

Walsh, M. A., Dementieva, I., Evans, G., Sanishvili, R. \& Joachimiak, A. (1999). Acta Cryst. D55, 1168-1173.

Walsh, M. A, Otwinowski, Z., Perrakis, A., Anderson, P. M. \& Joachimiak, A. (1999). In preparation.

Westbrook, E. M. \& Naday, I. (1997). Methods Enzymol. 276, 233-243.

Zhang, R., Evans, G., Rotella, F. J., Westbrook, E. M., Beno, D., Huberman, E., Joachimiak, A. \& Collart, F. R. (1999). Biochemistry, 38, 4691-4700. 